\title{
Co-ultraPEALut Effect on Mild Cognitive Impairment: A Retrospective Observational Study
}

\section{Barbara Manni ${ }^{1 *}$, Lucia Federzoni' ${ }^{2}$, Patrizia Zucchi ${ }^{1}$ and Andrea Fabbo $^{1}$}

${ }^{1}$ Cognitive Disorders and Dementia Unit, Primary Care Department, Local Agency

for Health (AUSL) of Modena, Italy

${ }^{2}$ School of Geriatrics, University of Modena and Reggio Emilia, Modena, Italy

*Corresponding Author: Barbara Manni, Cognitive Disorders and Dementia Unit, Primary Care Department, Local Agency for Health (AUSL) of Modena, Italy.
Received: April 15, 2021

Published: June 04, 2021

(C) All rights are reserved by Barbara Manni., et al.

\begin{abstract}
Mild cognitive disorder (MCI) may precede the onset of cognitive decline. Neuroinflammation may have a leading role in early phase of many neurocognitive diseases and may represent a useful target to limit MCI progression. Palmitoylethanolamide is endowed with anti-inflammatory and neuroprotective activities. Its formulation containing luteolin (PEALut) has been found to improve memory impairments in experimental models and in cognitively impaired patients suggesting its potential use for delaying the dementia onset. This retrospective study provides a preliminary evaluation of PEALut effect on clinical aspects in MCI subjects. Data related to three MCI subject groups, matched case control for age, sex and comorbidity, were obtained via retrospective chart review. Groups received PEALut, dietary food and no treatment, respectively. Mini-Mental State Examination (MMSE) questionnaire was used to evaluate cognitive symptoms; Activity Daily Living and Instrumental Activity functional Daily Living were used for measuring activity daily living and functional status; UCLA-Neuropsychiatric Inventory was used for behavioral symptoms. All evaluations were performed at the baseline and after 6 months. PEALut group showed a significant improvement in short-term memory MMSE domain. Non cognitive behavioral disturbs were also improved in PEALut group. This latter effect seems to be limited to nonamnestic MCI patients. The data reported add another piece of the framework of evidence supporting the ability of PEA, or its composites, to slow down the progression of diseases of the central nervous system, and encourage to perform a double-blind clinical study to deepen PEALut effects on cognitive and non cognitive alterations.
\end{abstract}

Keywords: Dementia; Mild Cognitive Disorder; Neuropsychiatric Symptoms; PEALut

\section{Introduction}

Mild cognitive disorder, also named mild cognitive impairment (MCI), is defined as a slight alteration in cognitive function with otherwise normal function in the performance of daily living (Diagnostic and Statistical Manual of Mental Disorders, DSM-5). These criteria select subjects with a normal cognitive functions but amnesic functions similar to those of subjects with mild Alzheimer disease (AD) [1]. Longitudinal studies have shown that these subjects have a higher risk of developing $\mathrm{AD}$, whose estimate ranges from 10 to $50 \%$, based on the clinical criteria and time of follow up [2-5]. Subjects with MCI often present neuropsychiatric symptoms; in particular, apathy and depression have been suggested to be key indicators for progression to AD [6]. MCI may either be symptomatic of normal aging or a transition to early $\mathrm{AD}$ or other dementias [7]. MCI subjects are usually classified as amnestic MCI (aMCI) when the memory loss is predominant and it is associated 
with high risk to further conversion to AD [7] and nonamnestic MCI (non aMCI) when impairments are present in other domains than memory and have a higher risk to convert to other dementia forms such as diffuse Lewy body dementia.

To date, there are no specific treatments for $\mathrm{AD}$, the progressive and multifaceted degenerative phenotype suggests that new successful treatment strategies need to be equally multi-faceted and stage-specific. Given the poor efficacy of available treatments for overt dementia [8], much effort has being directed to identify strategies for limiting MCI progression, given the potential of such interventions to prevent/delay dementia onset. Growing evidence point out a lack of control of neuroinflammation as a leading actor in neurodegeneration and dementia onset, suggesting that the cell populations which orchestrate it (e.g. microglia, astrocytes, mast cells) may represent new promising therapeutic targets for limiting MCI progression [9-12].

Palmitoylethanolamide (PEA) is an endogenous lipid mediator used in clinic practice for its neuroprotective, antineuroinflammatory and pain relieving properties [13-15].

In particular, PEA reduced the cognitive impairments in an $\mathrm{AD}$ experimental model based on $A \beta_{25-35}$ peptide intracerebral injection [16]. More recently, beneficial PEA effects on both cognitive and other non-cognitive impairments have been demonstrated in 3xTg-AD mice, at two different stages of the pathology [17]. PEA, in its ultramicromized form ( $\mu \mathrm{mPEA}$ ), rescued cognitive functions in 6-month-old 3xTg-AD mice such as short- and long-term memory, contextual learning and memory, and spatial learning. In 12-month-old 3xTg-AD mice, PEA significantly improved the shortterm memory, with no significant effects on long-term memory.

Among formulations containing $\mu \mathrm{mPEA}$, the co-ultramicronized composite PEALut also contains luteolin (Lut) [18]. Lut is an important member of flavonoid family and exhibits strongly anti-inflammatory, antioxidant and phytoestrogen-like activities. An anti-amnesic and protective effect of Lut against $A \beta_{25-35^{-}}$ induced toxicity in mice has been reported [19], beside studies demonstrating that Lut modulates processes of synaptic plasticity, protects synapses from detrimental effects of chronic cerebral hypoperfusion in rats, which may contribute to protective effects of Lut on learning and memory as in neurodegenerative disorders like AD [20].
A recent study reported the case of an aMCI patient, who was treated for 9 months with high-dose PEALut, with a significant improvement of cognitive performances, documented by a $99 \mathrm{mTc}$ HMPAO single-photon emission computed tomography (SPECT) [21]. The positive PEA or PEALut effects on cognitive functions have been confirmed in clinical trials with different primary endpoints [22-24].

Given these evidence, our retrospective study aims to provide a preliminary evaluation of the effect of PEALut on clinical aspects of MCI subjects compared to non treaded-MCI controls or MCI receiving a supplementary food.

\section{Patients and Methods}

Data source and subjects

Data relative to eighty subjects (45 female and 35 male) with diagnosis of MCI made at the Cognitive Disorders and Dementia Unit, Primary Care Department, Local Agency for Health (AUSL) of Modena, during 2017, were obtained via retrospective chart review.

Subjects meeting DSM-5 criteria for mild cognitive disorder (MCI) were included in the study.

Exclusion criteria included the presence of major depressive disorder and diagnosis of major cognitive disorder (DSM-5).

The MCI subjects who freely underwent PEALut (group A), dietary supplement (group B) or none (group C) treatments were selected for this study. Group A received 700+70 mg/die microgranules PEALut for the first month followed by PEALut tablets, $400+40 \mathrm{mg} /$ die. Group B received a dietary supplement based on Choline, Homotaurin, vitamin B12 and vitamin E at the doses suggested by the producers.

The use of clinical data of subjects with diagnosis of MCI performed at the Cognitive Disorders and Dementia Unit, Primary Care Department, Local Agency for Health (AUSL) of Modena obtained via retrospective chart review was approved by the director of complex operating unit dated March 3, 2018 and an informed consent of all participants was obtained in accordance with guidelines of the Helsinki Declaration. 
Cognitive functions, daily living and functional status evaluations

Mini-Mental State Examination (MMSE) corrected for age and education was used to evaluate cognitive functions, Activity Daily Living (ADL) and Instrumental Activity functional Daily Living (IADL) were used for activity daily living and functional status.

\section{Non cognitive functions evaluations}

UCLA-Neuropsychiatric Inventory (NPI) was used to assess behavioral symptoms.

\section{Data analysis}

All evaluations were performed at the baseline (T0) and after 6 months (T1). Statistical analyses were performed with SPSS statistical package. Descriptive statistics (means and standard deviations) were calculated for each of the cognitive and non-cognitive measures at each time point. Total score related to cognitive and non-cognitive questionnaires was evaluated. For MMSE, the total score of selected items was also performed. Quantitative differences between groups (MMSE, ADL, IADL, NPI) were analyzed by ANOVA, followed by LSD post hoc analysis. Paired t-test was used to measure differences in indices between T0 and T6. Chi-square was used to compare qualitative differences (sex) between groups. $\mathrm{p}$ values $<0.05$ were considered statistically significant.

\section{Results}

Data from 80 MCI subjects of which 45 females and 35 male were analyzed. At baseline, subject groups did not differ for sex and age (Table 1. Chi-square test). Cognitive impairment was equal in groups $\mathrm{A}, \mathrm{B}$ and $\mathrm{C}$, all presented slight alteration in cognitive functions and normal functions in the performance of daily living activities (Table 1). ADL and NPI total scores were significantly different in the group $\mathrm{C}$ as compared to groups A and B (ANOVA followed by LSD post hoc analysis).

To compare cognitive and non-cognitive symptoms in MCI subject groups receiving PEALut, dietary supplement or no treatment, the difference of scores achieved at the end of treatments (6 months) minus baseline values ( $\Delta=\mathrm{T} 1-\mathrm{T} 0$ ) was determined. MMSE, IADL, ADL and NPI $\Delta$ mean values analyzed with one way ANOVA followed by LSD post hoc analysis showed no significant group differences (Table 2). However, when the values related to some MMSE items (those that in our experience are more sensi-

\begin{tabular}{|l|c|c|c|c|}
\hline & \multicolumn{3}{|c|}{ Groups } & p \\
\hline & A & B & C & \\
\hline n (F/M) & $35(16 / 19)$ & $21(12 / 9)$ & $24(17 / 7)$ & 0.160 \\
\hline Age & $80.9 \pm 6.3$ & $82.1 \pm 5.9$ & $82.2 \pm 3.9$ & 0.581 \\
\hline MMSE & $24.2 \pm 3.3$ & $25.3 \pm 2.4$ & $24.2 \pm 3.1$ & 0.359 \\
\hline ADL & $5.7 \pm 0.5$ & $5.3 \pm 1.1$ & $4.9 \pm 1.3$ & 0.005 \\
\hline IADL & $5.4 \pm 1.5$ & $6.1 \pm 2.2$ & $4.8 \pm 2.2$ & 0.084 \\
\hline NPI & $10.1 \pm 7.4$ & $8.0 \pm 6.9$ & $15.7 \pm 12.4$ & 0.013 \\
\hline
\end{tabular}

Table 1: Demographic and clinical characteristics of the studied groups at baseline examination. A, B, C = PEALut, dietary supplement and MCI control group, respectively. Data are expressed as mean scores \pm SD. Chi square test was used to compare gender between groups, one way ANOVA for the other parameters.

tive in clinical impairment) were analyzed with t-test for paired sample, a significant improvement of short memory was observed in subjects belonging to the group A who received PEALut treatment (Table 3).

\begin{tabular}{|l|c|c|c|c|}
\hline & \multicolumn{3}{|c|}{ Groups } & p \\
\hline & A & B & C & \\
\hline MMSE & $0.0 \pm 2.6$ & $0.2 \pm 2.9$ & $-0.6 \pm 3.0$ & 0,691 \\
\hline ADL & $-0.2 \pm 0.5$ & $0.1 \pm 7.0$ & $0.0 \pm 0.6$ & 0,161 \\
\hline IADL & $-0.2 \pm 1.1$ & $-0.8 \pm 1.5$ & $-0.2 \pm 0.9$ & 0,119 \\
\hline NPI & $-3.2 \pm 7.1$ & $-2.4 \pm 6.9$ & $-3.3 \pm 12.1$ & 0,937 \\
\hline
\end{tabular}

Table 2: MMSE, IADL, ADL and NPI $\Delta$ mean values in the analyzed groups. A, B, C = PEALut, dietary supplement and MCI control group, respectively. Data are the mean \pm SD evaluated as delta of T1-T0 scores. Group A: $\mathrm{n}=35$; group B: $\mathrm{n}=21$; group C: $\mathrm{n}=24$. Oneway ANOVA was used to obtained p values.

Moreover, MCI subjects treated with PEALut showed a significant improvement of neuropsychiatric symptoms at the end of the treatment, in fact, the NPI total score goes from $10.1 \pm 7.4$ at baseline to $7.0 \pm 6.6$ after six months treatment $(\mathrm{p}=0.011, t$-test for paired sample Table 4). When aMCI and non aMCI subjects were analyzed separately, the PEALut effect was confirmed in non aMCI subjects but not in aMCI. No change in NPI total score was observed in groups that received dietary supplement (B) or no treatment (C; Table 5). 


\begin{tabular}{|l|c|c|c|c|c|c|c|c|c|}
\hline \multicolumn{1}{|c|}{ MMSE item } & \multicolumn{3}{|c|}{ A } & \multicolumn{2}{c|}{ B } & \multicolumn{3}{c|}{ C } \\
\hline & T0 & T1 & p & T0 & T1 & p & T0 & T1 & p \\
\hline Orientation & $4.0 \pm 1.4$ & $3.8 \pm 1.8$ & $>0.05$ & $4.1 \pm 1.3$ & $4.1 \pm 1.4$ & $>0.05$ & $3.8 \pm 1.1$ & $3.7 \pm 1.3$ & $>0.05$ \\
\hline Attention & $3.2 \pm 1.8$ & $3.0 \pm 1.8$ & $>0.05$ & $3.2 \pm 1.6$ & $3.1 \pm 1.7$ & $>0.05$ & $2.8 \pm 2.0$ & $2.7 \pm 1.7$ & $>0.05$ \\
\hline Short Memory & $1.3 \pm 1.0$ & $1.7 \pm 1.1$ & 0.021 & $1.8 \pm 1.1$ & $2.0 \pm 1.1$ & $>0.05$ & $2.0 \pm 1.0$ & $2.0 \pm 1.1$ & $>0.05$ \\
\hline Praxia & $0.6 \pm 0.5$ & $0.7 \pm 0.5$ & $>0.05$ & $0.8 \pm 0.4$ & $0.76 \pm 0.4$ & $>0.05$ & $0.5 \pm 0.5$ & $0.5 \pm 0.5$ & $>0.05$ \\
\hline
\end{tabular}

Table 3: Mean scores of some MMSE items in subjects who received PEALut (A), dietary supplement (B) or no treatment (C). Data are expressed as mean scores \pm SD; T0 = baseline; T1 = 6 months. Group A n = 35; group B n = 21; group C $n=24$. T-test for paired samples was used to obtained p values.

\begin{tabular}{|l|c|c|c|c|}
\hline Group & $\mathbf{n}$ & T0 & T1 & p \\
\hline A & 35 & $10.1 \pm 7.4$ & $7.0 \pm 6.6$ & 0.011 \\
\hline B & 21 & $8.0 \pm 6.9$ & $5.5 \pm 5.8$ & $>0.05$ \\
\hline C & 24 & $15.7 \pm 12.4$ & $12.4 \pm 9.6$ & $>0.05$ \\
\hline
\end{tabular}

Table 4: Mean scores of total NPI in MCI subjects who received PEALut (A), dietary supplement (B), no treatment (C). Data are expressed as mean scores $\pm \mathrm{SD}$; $\mathrm{T} 0=$ baseline; $\mathrm{T} 1=6$ months. t-test for paired samples was used to obtained $p$ values.

\begin{tabular}{|l|c|c|c|c|}
\hline \multicolumn{1}{|c|}{ Group } & n & T0 & T1 & p \\
\hline aMCI & 12 & $8.7 \pm 7.3$ & $6.7 \pm 7.0$ & $>0.05$ \\
\hline non aMCI & 23 & $12.2 \pm 10.0$ & $8.7 \pm 8.2$ & 0.007 \\
\hline
\end{tabular}

Table 5: Mean scores of total NPI in aMCI and non aMCI subjects who received PEALut (A), dietary supplement (B) or no treatment (C). Data are expressed as mean scores $\pm \mathrm{SD}$; T0 = baseline; $\mathrm{T} 1=6$ months. T-test for paired samples was used to obtained $\mathrm{p}$ values.

None of the participants reported side effects attributable to PEALut or to food supplement intake.

\section{Discussion and Conclusion}

The increase in the older people population together with that of $\mathrm{AD}$ and other types of dementia constitute a dramatic challenge for public health services. The enormous social impact and the lack of effective pharmacological treatments make the research of ways to exploit and maximize the cognitive resources of subjects with initial symptoms of dementia of most importance to reduce or slow down the onset of AD and other dementias.

The results of this retrospective study show a significant improvement of short memory in MCI subjects who had taken PEALut for six months. These subjects also showed a significant improvement of neuropsychiatric symptoms.

Our results give support to a recent case report [21] described PEALut benefit in a 67-years elder affected by aMCI who showed a significant improvement of cognitive performances besides an almost perfusion SPECT normalization after 9 months treatment. A PEALut-induced improvement of cognitive functions has been reported in older people with mood disorders [24] as well as in sub-acute/chronic post-stroke patients [22].

The finding that we observed an effect only on short-term memory and not on total MMSE score may likely be due to low severity of cognitive impairments in the study population. Even if small, improvement of short-term memory is clinically relevant, and it is supported by analogous results obtained in different experimental models [17,25-26]. Short-term memory is among the first cognitive symptoms that occur in MCI [27], the finding that PEALut counteracts it suggests that the composite could slow down the progression of the symptoms.

Beside cognitive, behavioral and psychological symptoms are common in MCI and are associated with a higher risk of dementia [28]. It has been recently reported that NPI symptoms are frequent in MCI when compared to cognitively normal. Higher odds for delusions, hallucinations, disinhibition and psychomotor alterations have been reported when comparing MCI subjects with those who 
were cognitively normal [6]. Our study shows that PEALut significantly reduced non cognitive symptoms (NPI) present in MCI. The relatively higher, and non statistically significant, deficits found in non aMCI vs aMCI may explain the difference in the response to PEALut. A significant amelioration in the behavioral symptoms, as measured by changes from baseline NPI mean score, has been also recently observed in patients affected by frontotemporal dementia after 4 weeks treatment with PEALut. Importantly, this effect was paralleled by an improvement in frontal executive functions as revealed by an improvement in the Frontal Assessment Battery score [29]. These evidence suggest that PEALut can act both on the cognitive components and on non-cognitive symptoms in patients with dementia or those that risk to develop it.

Neither our data nor those reported in patients with dementia provide indications on how PEALut induces its effects in cognitively impaired patients. It is now known that PEA can act via multiple mechanisms. The first mechanism of action for PEA was proposed by Rita Levi-Montalcini's research group, who suggested that PEA acts via 'Autacoid Local Injury Antagonism (ALIA)' to downregulate mast cell activation [30,31]. Later, the existence of a direct receptor-mediated mechanism was proposed, and several studies demonstrated that PEA can act on many non-neuronal cells [32] (i.e. mast cell, microglia, astrocytes, oligodendrocytes) via direct activation of at least two different receptors: the PPAR- $\alpha$ [33] and the orphan GPCR55 [34]. The final action of PEA is the control of neuroinflammation with consequent neuroprotection and pain relief. Since some authors have suggested a correlation between neuropsychiatric symptoms and pro/anti-inflammatory equilibrium in cerebrospinal fluid [35], it is likely that the PEALut effect on behavioral and psychological symptoms might be ascribed to the control of neuroinflammation.

The data reported in the present study add another piece of the framework of evidence supporting the ability of PEA or its composites to slow down the progression of diseases of the central nervous system, as shown for Parkinson's disease [36]. Amyotrophic Lateral Sclerosis [37], mood disorders [38], autism [39] and postoperative delirium [40].

The main limitations of the present results include the nonrandomized study design and the small participants number. In addition, the low disturbance thresholds at baseline makes behavioral domain limited in its weight. However, the reported data encourage to perform a larger, randomized study to validate PEALut as a strategy for limiting MCI progression. In particular, it would be appropriate to evaluate a higher dosage of PEALut, in order to test a dosages comparable to those used in other studies $[22,24]$.

Further studies would be desirable also considering both the safety of the product and the urgency of characterizing innovative products for limiting MCI progression.

\section{Bibliography}

1. RC Petersen., et al. "Mild cognitive impairment: clinical characterization and outcome". Archives of neurology 56.3 (1999): 303-308.

2. A Bozoky., et al. "Mild cognitive impairments predict dementia in non demented elderly patients with memory loss". Archives of neurology 58 (2001): 411-416.

3. RC Petersen. "Normal aging, mild cognitive impairment, and early Alzheimer's disease”. Neurologist 1 (1995): 326-344.

4. SE Black. "Can SPECT predict the future for mild cognitive impairment?". Canadian Journal of Neurological Sciences 26 (1999): 4-6.

5. K Ritchie and J Touchon. "Mild cognitive impairment: conceptual basis and current nosological status". Lancet 355 (2000): 225-228.

6. PV Nunes., et al. "Neuropsychiatric Inventory in CommunityDwelling Older Adults with Mild Cognitive Impairment and Dementia”. Journal of Alzheimer's Disease 68.2 (2019): 669678.

7. M Grundman., et al. "Mild cognitive impairment can be distinguished from Alzheimer disease and normal aging for clinical trials". Archives of Neurology 61.1 (2004): 59-66.

8. PJ Ousset., et al. "Is Alzheimer's Disease Drug Development Broken? What Must Be Improved". The Journal of Prevention of Alzheimer's Disease 1.1 (2014): 40-45.

9. D Kempuraj., et al. "Mast Cell Activation in Brain Injury, Stress, and Post-traumatic Stress Disorder and Alzheimer's Disease 
Pathogenesis". Frontiers in Neuroscience 11 (2017): 703.

10. KA Clayton., et al. “Alzheimer's Disease: The Role of Microglia in Brain Homeostasis and Proteopathy". Frontiers in Neuroscience 11 (2017): 680.

11. RE González-Reyes., et al. "Involvement of Astrocytes in Alzheimer's Disease from a Neuroinflammatory and Oxidative Stress Perspective". Frontiers in Molecular Neuroscience 10 (2017): 427.

12. NH Zawia., et al. "Epigenetics, oxidative stress, and Alzheimer disease”. Free Radical Biology and Medicine 46 (2009): 12411249.

13. E Esposito and S Cuzzocrea. "Palmitoylethanolamide in homeostatic and traumatic central nervous system injuries". CNS and Neurological Disorders - Drug Targets 12.1 (2013): $55-61$.

14. G MattaceRaso., et al. "Palmitoylethanolamide in CNS health and disease". Pharmacological Research 86 (2014): 32-41.

15. SD Skaper., et al. "N-Palmitoylethanolamine and neuroinflammation: a novel therapeutic strategy of resolution". Molecular Neurobiology 52.2 (2015): 1034-1042.

16. G D'Agostino., et al. "Palmitoylethanolamide protects against the amyloid- $\beta 25$-35-induced learning and memory impairment in mice, an experimental model of Alzheimer disease". Neuropsychopharmacology 37 (2012): 1784-1792.

17. C Scuderi., et al. "Ultramicronized palmitoylethanolamide rescues learning and memory impairments in a triple transgenic mouse model of Alzheimer's disease by exerting anti-inflammatory and neuroprotective effects". Translational Psychiatry 8.1 (2018): 32.

18. R Crupi., et al. "Effects of palmitoylethanolamide and luteolin in an animal model of anxiety/depression". CNS and Neurological Disorders - Drug Targets 12.7 (2013): 989-1001.

19. R Liu., et al. "The anti-amnestic effects of Luteolin against amyloid-25-35 peptide-induced toxicity in mice involve the protection of neuromuscular unit". Neuroscience 162 (2009): 1232-1243.
20. B Xu., et al. "Luteolin promotes long-term potentiation and improves cognitive functions in chronic cerebral hypoperfused rats". European Journal of Pharmacology 627.1-3 (2010): 99105.

21. RS Calabrò., et al. "PEALut efficacy in mild cognitive impairment: evidence from a SPECT case study!". Aging Clinical and Experimental Research 28.6 (2016): 1279-1282.

22. C Caltagirone., et al. "Co-ultramicronized palmitoylethanolamide/luteolin in the treatment of cerebral ischemia: from rodent to man". Translational Stroke Research 7.1 (2016): 54-69.

23. NS Orefice., et al. "Oral palmitoylethanolamide treatment is associated with reduced cutaneous adverse effects of interferon- $\beta 1 \mathrm{a}$ and circulating proinflammatory cytokines in relapsing-remitting multiple sclerosis". Neurotherapeutics 13.2 (2016): 428-438.

24. V Uva. "Palmitoiletanolamide e Luteolina coultramicronizzate (co ultraPEALut) nel trattamento dei disturbi dell'umore nell'anziano". Psicogeriatria 1 (2017): 79-87.

25. S Boccella., et al. "Metabotropic Glutamate Receptor 5 and 8 Modulate the Ameliorative Effect of Ultramicronized Palmitoylethanolamide on Cognitive Decline Associated with Neuropathic Pain". International Journal of Molecular Sciences 20.7 (2019).

26. MI Holubiec., et al. "Palmitoylethanolamide prevents neuroinflammation, reduces astrogliosis and preserves recognition and spatial memory following induction of neonatal anoxia-ischemia". Psychopharmacology (Berl) 235.10 (2018): 2929-2945.

27. RC Petersen., et al. "Mild cognitive impairment: a concept in evolution". Journal of Internal Medicine 275.3 (2014): 214-228.

28. MA Sugarman., et al. "Neuropsychiatric symptoms and the diagnostic stability of Mild Cognitive Impairment". Journal of Alzheimer's Disease 62.4 (2018): 1841-1855.

29. M Assogna., et al. "A possible role of palmitoylethanolamide combined with luteoline in frontotemporal dementia treatment: a clinical and neurophysiological study". European 
Journal of Neurology 26.1 (2019): 112-346.

30. R Levi-Montalcini., et al. "Nerve growth factor: from neurotrophin to neurokine". Trends in Neuroscience 19 (1996): 514-520.

31. L Aloe., et al. "A proposed autacoid mechanism controlling mastocyte behavior". Agents Actions 39 (1993): 145-147.

32. SD Skaper., et al. "An Inflammation-Centric View of Neurological Disease: Beyond the Neuron". Frontiers in Cellular Neuroscience 12 (2018): 72.

33. J Lo Verme., et al. "The nuclear receptor peroxisome proliferator-activated receptor-alpha mediates the antiinflammatory actions of palmitoylethanolamide". Molecular Pharmacology 67 (2005): 15-19.

34. E Ryberg., et al. "The orphan receptor GPR55 is a novel cannabinoid receptor". British Journal of Pharmacology 152 (2007): 1092-1101.

35. S Holmgren., et al. "Neuropsychiatric symptoms in dementia-a role for neuroinflammation?". Brain Research Bulletin 108 (2014): 88-93.

36. S Brotini., et al. "Ultra-micronized Palmitoylethanolamide: An Efficacious Adjuvant Therapy for Parkinson's Disease". CNS and Neurological Disorders - Drug Targets 16.6 (2017): 705713.

37. E Palma., et al. "Acetylcholine receptors from human muscle as pharmacological targets for ALS therapy". Proceedings of the National Academy of Sciences 113.11 (2016): 3060-3065.

38. M Ghazizadeh-Hashemi., et al. "Palmitoylethanolamide as adjunctive therapy in major depressive disorder: A doubleblind, randomized and placebo-controlled trial". Journal of Affective Disorders 232 (2018): 127-133.

39. M Khalaj., et al. "Palmitoylethanolamide as adjunctive therapy for autism: Efficacy and safety results from a randomized controlled trial". Journal of Psychiatric Research 103 (2018): 104-111.
40. ML Lunardelli., et al. "Co-ultraPEALut: Role in Preclinical and Clinical Delirium Manifestations". CNS and Neurological Disorders - Drug Targets 18.7 (2019): 530-554.

\section{Volume 4 Issue 10 October 2021}

(c) All rights are reserved by Barbara Manni., et al. 